\title{
Mid-wave infrared InAs/GaSb type-II superlattice photodetector with n-B-p design grown on GaAs substrate
}

\author{
Zhuo Deng *, Daqian Guo *, Jian Huang, Huiyun Liu, Jiang Wu, and Baile Chen
}

\begin{abstract}
In this work, we report the direct growth and characterization of a mid-wave infrared InAs/GaSb type-II superlattice n-B-p photodetector on GaAs substrate. The design consists of an n-doped contact, a wide bandgap unipolar barrier and a p-doped absorber, which uses photogenerated electron as minority carriers to enjoy the longer electron diffusion length as compared to hole diffusion length. At $77 \mathrm{~K}$, the device exhibits a dark current density of $2.9 \times 10^{-5} \mathrm{~A} / \mathrm{cm}^{2}$ under $-0.1 \mathrm{~V}$, and a zero-bias differential-resistance-area product $\left(R_{0} A\right)$ in excess of $8 \times 10^{3} \Omega \cdot \mathrm{cm}^{2}$. Arrhenius analysis of dark current demonstrates that the dominant mechanism is diffusion at temperature higher than $130 \mathrm{~K} .50 \%$ cutoff wavelength of the detector is found at 6.4 $\mu \mathrm{m}$ at $77 \mathrm{~K}$ under zero bias, with a peak responsivity of $0.56 \mathrm{~A} / \mathrm{W}$. The corresponding specific detectivity is $7.6 \times 10^{11} \mathrm{~cm} \cdot \mathrm{Hz} z^{1 / 2} / \mathrm{W}$. Key device parameters which limit the further optimization of performance are discussed.
\end{abstract}

Index Terms - GaAs substrate, InAs/GaSb type-II superlattice, Mid-wave infrared, Photodetector

\section{INTRODUCTION}

$I^{1}$ $n$ the past two decades, mid-wave infrared (MWIR) photodetectors based on InAs/GaSb type-II superlattice (T2SL) has been the subject of extensive investigation as a competitive alternative to other detector technologies, such as $\mathrm{HgCdTe}$ (MCT), quantum wells and quantum dots, in both civil and military application sectors [1-3]. Flexibility of tailoring the detection wavelength by changing the thickness and composition of constituent layers [4], longer Auger recombination lifetime [5] and larger electron effective mass [6] have been reported in the InAs/GaSb T2SL system. These advantages have enabled the fabrication of high-performance

This work was supported in part by the Shanghai Sailing Program under Grant 17 YF1429300, in part by the ShanghaiTech University startup funding under Grant F-0203-16-002, in part by the UK EPSRC First Grant EP/R006172/1. (Corresponding authors: Jiang Wu and Baile Chen.)

Z. Deng, J. Huang and B. Chen are with the Optoelectronic Device Laboratory, School of Information Science and Technology, ShanghaiTech University, Shanghai 201210, Peoples R China (e-mail: dengzhuo@ shanghaitech.edu.cn; $\quad$ huangiian@shanghaitech.edu.cn; chenbl@shanghaitech.edu.cn).

D. Guo, H. Liu and J. Wu are with the Department of Electronic and Electrical Engineering, University College London, London WC1E 7JE, United Kingdom (e-mail: daqian.guo.15@ucl.ac.uk; huiyun.liu@ucl.ac.uk; jiang.wu@ucl.ac.uk).

J. Wu present address: Institute of Fundamental and Frontier Sciences, University of Electronic Science and Technology of China, Chengdu 610054 Sichuan, Peoples R China

*These authors contributed equally to this work. focal plane arrays (FPAs) based on T2SL to accommodate the application needs covering from the mid-wave to long-wave infrared region $[3,7]$. Nevertheless, the significant progress of InAs/GaSb T2SL technology is mainly limited by two constraints. First, multiple dark current mechanisms including diffusion, generation-recombination (G-R) associated with the Shockley-Read-Hall (SRH) processes, tunneling and surface leakage would degrade the device performance in conventional $\mathrm{p}-\mathrm{i}-\mathrm{n}$ architecture. To address these problems, various heterostructures such as n-B-n [6], n-B-p [8], and complementary p-B-i-B-n [9] designs which utilize the engineering freedom of band structure of T2SL have been demonstrated in order to alleviate different dark current component. The second factor, which limits the realization of large format T2SL based FPAs, is the lack of low cost and large area substrates. Traditionally the T2SL detectors with superior performance are only grown on lattice-matched GaSb substrate, which suffers from large absorption coefficient for infrared radiation beyond $5 \mu \mathrm{m}$ and shortage of supply with diameter larger than 4 inches in semiconductor industry [10]. As an alternative to grow T2SL detectors, GaAs substrate offers several advantages over the native GaSb substrate in terms of infrared transparency, size and cost [11]. However, formation of defects is inevitable while growing the GaSb layers on a highly mismatched $(\Delta a / a \sim 7.8 \%)$ GaAs substrate. One approach is to use interfacial misfit (IMF) array to completely relief the strain energy at the $\mathrm{GaSb} / \mathrm{GaAs}$ interface [12]. In the IMF growth mode, both $90^{\circ}$ and $60^{\circ}$ misfit dislocations (MDs) could present at the $\mathrm{GaSb} / \mathrm{GaAs}$ interface. The $90^{\circ} \mathrm{MDs}$ are pure edge type dislocations which propagate laterally along the [110] and [1-10] directions; the $60^{\circ}$ MDs can generate vertical propagated threading dislocations (TDs) along the (111) planes into the device region, which could contribute to high level of dark current $[13,14]$. By using the IMF growth mode, T2SL detectors demonstrated on the highly lattice mismatched substrates have shown promising results comparing with the counterparts grown on the native substrates $[10,13]$.

In this work, we report a MWIR InAs/GaSb T2SL photodetector with a unipolar barrier heterostructure, namely the n-B-p architecture on GaAs substrate. The device design consists of an n-type contact, an n-type wide bandgap barrier and a p-type absorber. Similar to the n-B-n design, in n-B-p structure most of the depletion electric field drops across the wide bandgap barrier layer which is designed to reduce the G-R component of dark current. On the other hand, unlike the n-B-n design, in n-B-p variant the $n$-barrier/p-absorber junction offers a built-in potential, which enables zero-bias operation of the 
device. In addition, in n-B-n design the photocurrent is based on minority holes, while in n-B-p structure the p-doped absorber enables the higher mobility electrons to be the minority photocarriers, which is capable to attain higher quantum efficiency. The device demonstrated in this study shows a $50 \%$ cutoff wavelength of $6.4 \mu \mathrm{m}$ at $77 \mathrm{~K}$ under zero bias. The corresponding quantum efficiency $(\mathrm{QE})$ at near-cutoff is between 10 to $15 \%$, and a peak specific detectivity of $7.6 \times 10^{11} \mathrm{~cm} \cdot \mathrm{Hz}^{1 / 2} / \mathrm{W}$ is obtained. These device parameters are significantly improved compared with the T2SL detector with conventional p-i-n design grown on GaAs substrate, but further optimizations are needed to achieve comparable performance of the devices on native GaSb substrate.

\section{EXPERIMENTAL DETAILS}

Schematic diagram of the InAs/GaSb T2SL n-B-p device structure is shown in Fig. 1 (a). The device layers were directly grown on a GaAs (001) substrate using a solid-source molecular beam epitaxy system. The growth was started with a $200 \mathrm{~nm}$ thick GaAs layer at $590{ }^{\circ} \mathrm{C}$ to smooth the GaAs surface. After closing the arsenic valve, the growth was interrupted for $5 \mathrm{~s}$ before opening the antimony valve. The growth of $\mathrm{GaSb}$ buffer was taken place at $480{ }^{\circ} \mathrm{C}$ with a growth rate of 0.7 monolayer (ML) per second. The n-B-p structure consists of a $500 \mathrm{~nm}$ thick $\mathrm{p}^{+}$-doped bottom contact layer formed by $10 \mathrm{ML}$ InAs:Be $\left(1.98 \times 10^{18} \mathrm{~cm}^{-3}\right) / 10 \mathrm{ML} \mathrm{GaSb}: \operatorname{Be}\left(1.1 \times 10^{18} \mathrm{~cm}^{-3}\right) \mathrm{SLs}$, followed by a $2000 \mathrm{~nm}$ thick p-doped $10 \mathrm{ML}$ InAs:Be $\left(1 \times 10^{17}\right.$ $\left.\mathrm{cm}^{-3}\right) / 10 \mathrm{ML}$ GaSb:Be $\left(4.9 \times 10^{16} \mathrm{~cm}^{-3}\right)$ SLs absorber layer. Then a $250 \mathrm{~nm}$ thick n-doped barrier layer made of $6 \mathrm{ML}$ $\mathrm{AlSb} / 12 \mathrm{ML}$ InAs:Si $\left(1 \times 10^{16} \mathrm{~cm}^{-3}\right)$ SLs was grown, followed by a $200 \mathrm{~nm}$ thick $\mathrm{n}^{+}$-doped $5 \mathrm{ML}$ InAs:Si $\left(1 \times 10^{18} \mathrm{~cm}^{-3}\right) / 5 \mathrm{ML}$ $\mathrm{GaSb}$ SLs top contact layer. Finally the structure was terminated by a $50 \mathrm{~nm}$ thick $\mathrm{n}^{+}$-doped InAs:Si $\left(1 \times 10^{18} \mathrm{~cm}^{-3}\right)$ cap layer to assure a good ohmic contact property. Fig. 1 (b) depicts the calculated energy band alignment of the T2SL $n$-B-p structure under zero bias. The wide bandgap barrier layer provides a large valence band offset and minimal conduction band offset. It is clear that most of the electric field drops across the $\mathrm{n}$-barrier/p-absorber junction while the absorber region remains flat, which helps to alleviate G-R dark current in the device. As mentioned previously, the p-type doping of the absorber layer is intended to utilize the higher mobility electrons as minority photogenerated carriers to improve the collection efficiency and thus quantum efficiency of device. It should be further noted that as a general design guideline, the optimal doping level for T2SL absorber is on the order of $10^{16}$ $\mathrm{cm}^{-3}$, considering the simultaneous impact of doping concentration on diffusion dark current and minority carrier lifetime [15]. For the n-B-p T2SL structure studied in this work, the $\mathrm{p}$-absorber was intentionally doped to a level of $\sim 5-10 \times 10^{16}$, aiming to reduce the diffusion current. However, one needs to take the Auger recombination effect into consideration when designing the absorber layer. For instance, Taghipour et al. have investigated the transport properties of minority carriers in a similar MWIR n-B-p T2SL detector grown on GaSb, in which an Auger-limited minority carrier lifetime of $\sim 20 \mathrm{~ns}$ and a corresponding diffusion length of $<5 \mu \mathrm{m}$ were reported for temperature higher than $150 \mathrm{~K}$ [16]. These results indicate that Auger recombination could be an important limiting factor to carrier transport when barrier-based T2SL detectors are operating at high temperature regime.

After the material growth, the wafer was processed into a set of mesa-isolated test devices with variable circular diameters using standard UV photo-lithographic processing technique. Mesa structures were defined by wet chemical etching with the solution $\mathrm{C}_{6} \mathrm{H}_{8} \mathrm{O}_{7}: \mathrm{H}_{3} \mathrm{PO}_{4}: \mathrm{H}_{2} \mathrm{O}_{2}: \mathrm{H}_{2} \mathrm{O} \quad(1: 1: 4: 16)$ and mesa surfaces were passivated by SU-8. Finally, metal contacts of $\mathrm{Ti} / \mathrm{Pt} / \mathrm{Au}(50 \mathrm{~nm} / 50 \mathrm{~nm} / 300 \mathrm{~nm})$ were formed at the top and bottom contacts by using e-beam evaporation and lift-off techniques. No anti-reflection coating was used in the device.

In order to probe the crystalline quality of the T2SL device structure, high-resolution X-ray diffraction (HRXRD) was performed on the as-grown device wafers using an X-ray diffractometer in double-crystal configuration. The sample was then cleaved and a test device with $130 \mu \mathrm{m}$ circular diameter was wire bonded. For electrical characterization, the device was loaded into a variable-temperature probe station and the dark current-bias voltage characteristics were analyzed by a semiconductor parameter analyzer. Afterwards the device was loaded into a variable-temperature cryostat for optical measurements. The relative photoresponse of the device was measured with a Fourier Transform Infrared Spectrometer in normal-incidence, top-illuminated configuration. In order to calibrate the spectral response and obtain the absolute responsivity of the device, a standard blackbody source operating at $700^{\circ} \mathrm{C}$ with chopper frequency of $130 \mathrm{~Hz}$ was used to illuminate the sample at different temperature. A low-noise current preamplifier was employed to apply external bias to the device and to record and amplify the output photocurrent simultaneously. The signal was then recorded by a lock-in amplifier with reference frequency fed by the optical chopper.

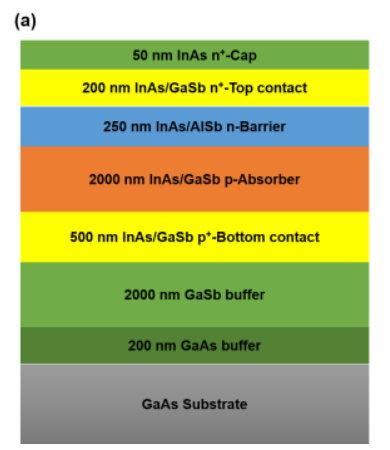

(b)

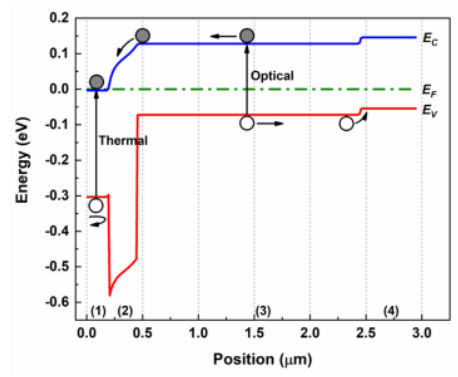

Fig. 1. (a) Schematic layout of the device structure; (b) Calculated energy band diagram of the T2SL device structure under zero bias. The layer sequence from left to right is: (1) $\mathrm{n}^{+}-$Top contact, (2) n-Barrier, (3) p-Absorber and (4) $\mathrm{p}^{+}$-Bottom contact. $E c, E v$ and $E_{F}$ represent conduction and valence band edge, and Fermi level respectively. Electrons are represented by grey circles while holes are drawn in hollow circles. The transitions associated with the photocurrent and dark current are labelled as "Optical" and "Thermal", respectively. 


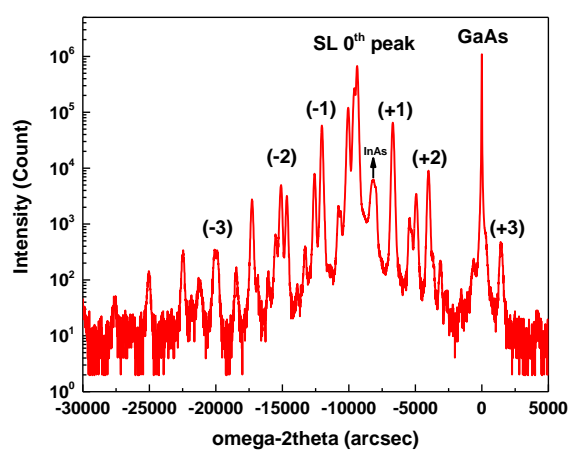

Fig. 2. HRXRD omega-2theta rocking curves measured from the T2SL as-grown device wafer.

\section{RESULTS AND DISCUSSIONS}

Fig. 2 shows the HRXRD omega-2theta rocking curves measured from the T2SL as-grown device wafer. Up to third-order satellite peaks for both the contacts and absorber superlattices are well defined in the sample. The sharp peak between the $\mathrm{SL}(0)$ and $\mathrm{SL}(+1)$ peaks is from the InAs cap layer. The Full-Width-at-Half-Maximum (FWHM) of the SL(0) and SL(+1) peaks are estimated to be $\sim 140$ and $\sim 150$ arcsec respectively. The FWHM of GaSb (004) peak is $\sim 135$ arcsec, which is comparable to the FWHM value mentioned in Ref. [12]. The dislocations density $\rho_{d}$ is estimated to be $\sim 5 \times 10^{7} \mathrm{~cm}^{-2}$, based on the equation $\rho_{d}=(\mathrm{FWHM} / 2 b)^{2}$, where the Burgers vector $b$ is $0.431 \mathrm{~nm}[17]$.

Dark current density-bias voltage characteristics measured from the T2SL device at different temperatures are presented in Fig. 3 (a). The test device has a circular diameter of $130 \mu \mathrm{m}$. It is noted that the dark current curves measured at $77 \mathrm{~K}$ and 100 $\mathrm{K}$ show small photovoltaic shifts to the positive bias, which are due to the imperfections in the cold shield of the probe station used in the measurements. At $77 \mathrm{~K}$, the device exhibits a dark current density of $2.9 \times 10^{-5} \mathrm{~A} / \mathrm{cm}^{2}$ under $-0.1 \mathrm{~V}$, and it increases to $5.8 \mathrm{~A} / \mathrm{cm}^{2}$ at room temperature. From the temperature dependent dark current curves, the differential-resistance-area product $(R A)$ as a function of bias at different temperatures can be evaluated, as plotted in Fig. 3 (b). The peak $R A$ at $77 \mathrm{~K}$ is found at $\sim 1.8 \times 10^{4} \Omega \cdot \mathrm{cm}^{2}$ under $-0.1 \mathrm{~V}$ bias, and the $R A$ under zero bias $\left(R_{0} A\right)$ is $\sim 8 \times 10^{3} \Omega \cdot \mathrm{cm}^{2}$. Fig. 4 shows the temperature dependent Arrhenius plot of the dark current density under applied bias of $-0.1 \mathrm{~V}$. Linear fit at low temperature regime (77-130 K) yields an activation energy $\left(E_{a}\right)$ of $\sim 61.6 \mathrm{meV}$, and at high temperature range (130-300 K) $E_{a}$ increases to $~ 159.9$ meV. Since the bandgap energy $\left(E_{g}\right)$ of the T2SL device varies from $\sim 184 \mathrm{meV}(77 \mathrm{~K})$ to $\sim 159 \mathrm{meV}(190 \mathrm{~K})$ as estimated from the absorption edge of the photoresponse spectra (see Fig. 5), at low temperature range $E_{a}=E_{g} / 3$, indicating that the device dark current is dominated by the G-R mechanism; at high temperature range $E_{a}=E_{g}$, suggesting that the device dark current is mainly contributed by diffusion.
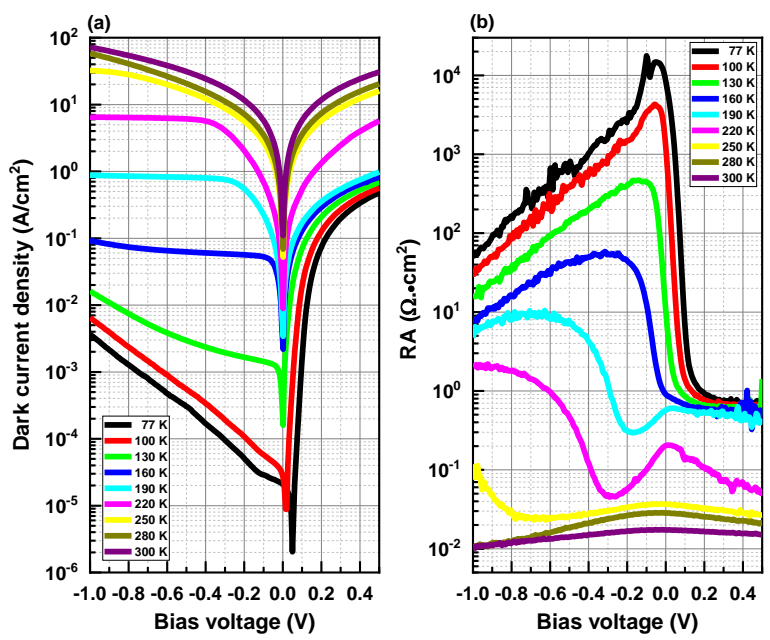

Fig. 3 (a) Temperature-dependent dark current density-voltage characteristics of the T2SL device with a circular diameter of $130 \mu \mathrm{m}$; (b) Differential-resistance-area product $(R A)$ as a function of bias voltage of the $\mathrm{T} 2 \mathrm{SL}$ device at different temperatures.

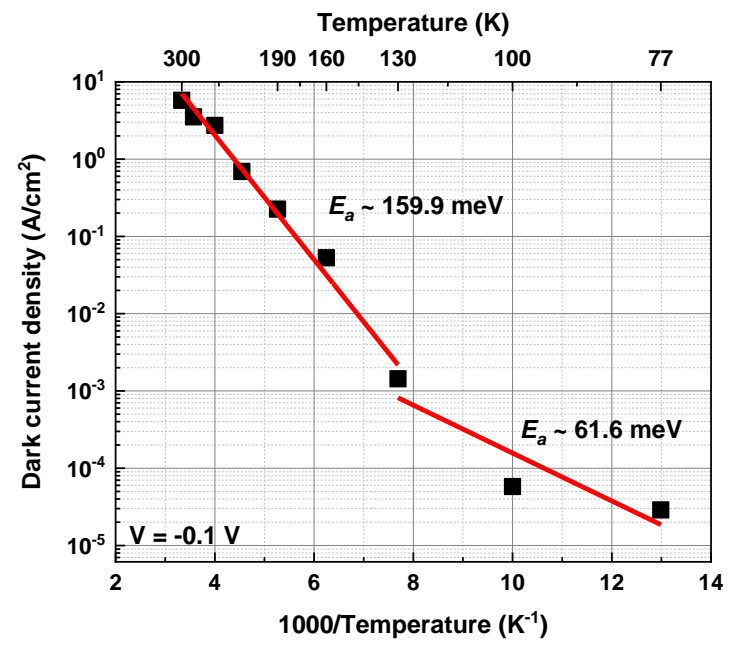

Fig. 4. Arrhenius plot of the dark current density under $-0.1 \mathrm{~V}$ bias. Solid lines represent the linear fit at different temperature regimes.

The photoresponse spectra of the T2SL device measured at different temperatures are shown in Fig. 5. At $77 \mathrm{~K}$ and under zero bias, the $50 \%$ cutoff wavelength $\left(\lambda_{50 \%}\right.$ cutoff $)$ is found at $\sim 6.4$ $\mu \mathrm{m}$, with a peak responsivity of $0.56 \mathrm{~A} / \mathrm{W}$. It shifts to $\sim 7.3 \mu \mathrm{m}$ at $190 \mathrm{~K}$, and the peak responsivity drops to $0.35 \mathrm{~A} / \mathrm{W}$. When temperature rises from 77 to $130 \mathrm{~K}$, the photoresponse slightly increases, which can partly be due to improved electron thermionic emission efficiency over the unexpected electron barrier induced in the conduction band, and partly be due to the increase of absorption coefficient with the red-shift of material bandgap, i.e., $\alpha(\lambda) \sim\left[E(\lambda)-E_{g}(T)\right]^{1 / 2}$. However further increase of temperature from $130 \mathrm{~K}$ onwards leads to a rapid drop in responsivity. It is believed this is due to the enhanced recombination rate of carriers at high temperature, thus the diffusion length is reduced and more and more carriers annihilate before they could reach the electrodes and contribute to the photocurrent[18]. Fig. 6 (a) depicts the quantum efficiency (QE) of the T2SL device at different temperatures. 
The oscillatory features observed before $4 \mu \mathrm{m}$ for all spectra, which exist originally in the photoresponse spectra, are due to the multiple reflections between the superlattice/air and the $\mathrm{GaSb} / \mathrm{GaAs}$ buffer interfaces as a consequence of different refractive indexes of the materials [19]. From 77 to $130 \mathrm{~K}$ the QE values at $4 \mu \mathrm{m}$ under zero bias stay around $15 \%$ until the temperature rises above $130 \mathrm{~K}$, as illustrated in Fig. 6 (b). A small bias of $-0.05 \mathrm{~V}$ is sufficient to recover the QE and fully extract the photogenerated carriers. Fig. 6 (c) shows the QE at $77 \mathrm{~K}$ at $4 \mu \mathrm{m}$ under different applied bias. It is clear that $\mathrm{QE}$ saturates at a bias beyond $-0.5 \mathrm{~V}$. This bias dependence of $\mathrm{QE}$ suggests that not all carriers are extracted at lower bias, which might be attributed to the presence of small unexpected conduction band offset. The QE value of our T2SL device is four times lower than that of the MWIR InAs/GaSb T2SL detector with similar n-B-p design on GaSb substrate [20], which could be due to the thinner $(2 \mu \mathrm{m}$ versus $4 \mu \mathrm{m})$ absorber and lower carrier lifetime in our GaAs based device. Additional works are required to increase the $\mathrm{QE}$ of our device by optimizing the crystal quality of absorber.

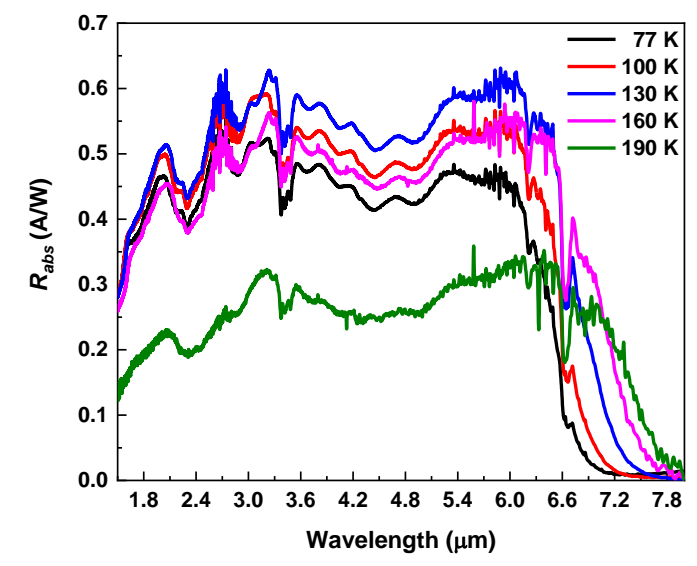

Fig. 5. Absolute responsivity $\left(R_{a b s}\right)$ of the T2SL device as a function of temperature under zero bias.
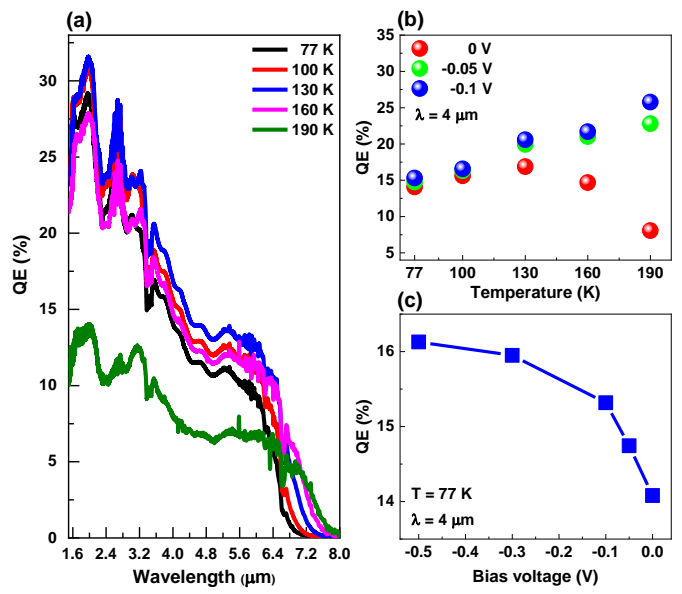

Fig. 6. Quantum efficiency (QE) of the T2SL device (a) at different temperatures under zero bias; (b) $\mathrm{QE}$ at $4 \mu \mathrm{m}$ at different temperatures under three different bias voltages; (c) $\mathrm{QE}$ at $77 \mathrm{~K}$ at $4 \mu \mathrm{m}$ as a function of bias.
Finally, the Johnson noise-limited specific detectivity $D^{*}$ of the T2SL device is calculated by[18, 21, 22]:

$$
D^{*}=R_{P} A^{\frac{1}{2}}\left(\frac{4 k_{B} T}{R_{0}}\right)^{-1 / 2}
$$

where $R_{p}$ is the peak responsivity, $A$ is the device mesa area, $k_{B}$ is Boltzmann's constant, $T$ is the temperature of the device, $R_{0}$ is the differential resistance under zero bias, and $q$ is the electron charge. Specific detectivity curves calculated at different temperatures are shown in Fig. 7. At $77 \mathrm{~K}$, a peak $D^{*}$ of $7.6 \times 10^{11} \mathrm{~cm} \cdot \mathrm{Hz}^{1 / 2} / \mathrm{W}$ is achieved under zero bias. This value is six times higher than the MWIR T2SL n-B-n counterpart [10], and an order of magnitude higher than that in the traditional p-i-n device grown on GaAs [23] (see TABLE 1), thanks to the much lower dark current density ( three order of magnitude) contributed by the barrier design. It is worth to note here, despite the additional SRH component, the dark current density in our device is about twenty times lower than the dark current density of the n-B-n on GaAs in Ref [10]. This can be attributed to lower defect density and the application of surface passivation layer. However, our device shows about an order of magnitude higher in dark current as compared with the similar n-B-p on native GaSb substrate. Thus, in order to achieve higher operating temperature and performance comparable with state-of-the-art MWIR T2SL device on native substrate, additional engineering of the present structure should be focused on optimization of defect density in the active layers to further reduce the device dark current, e.g., by growing more and/or thicker layers of dislocation buffer.

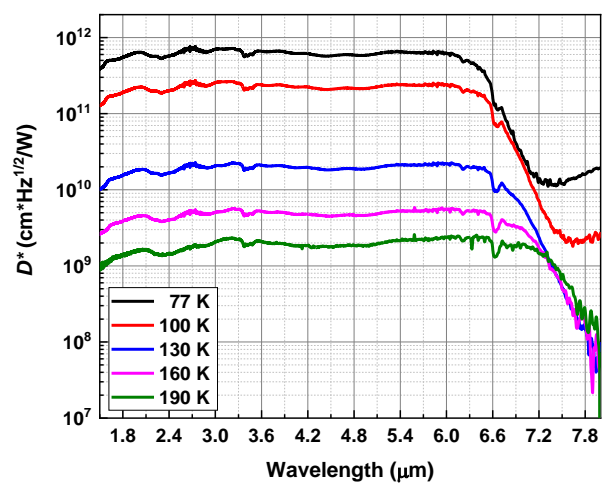

Fig. 7. Specific detectivity $\left(D^{*}\right)$ of the T2SL device at different temperatures.

TABLE 1. Comparison of $50 \%$ cutoff wavelength $\left(\lambda_{50 \%}\right.$ cutoff $)$, dark current density $\left(J_{d}\right)$ and peak detectivity $\left(D^{*}\right)$ of T2SL-based detectors grown on different substrates with various designs. Unless specified otherwise, all data shown are at $77 \mathrm{~K}$ and under zero bias.

\begin{tabular}{lllll}
\hline \hline $\begin{array}{l}\text { Device } \\
\text { parameters }\end{array}$ & n-B-p on & n-B-p on & n-B-n on & p-i-n on \\
$\lambda_{50 \% \text { cutoff }}(\mu \mathrm{m})$ & 6.4 & $5(0.2 \mathrm{~V})$ & $4.3(0.5 \mathrm{~V})$ & 4.3 \\
$J_{d}\left(0.1 \mathrm{~V}, \mathrm{~A} / \mathrm{cm}^{2}\right)$ & $2.9 \times 10^{-5}$ & $4.5 \times 10^{-6}$ & $6 \times 10^{-4}$ & $1 \times 10^{-2}$ \\
Peak $D^{*}$ (Jones) & $7.6 \times 10^{11}$ & N/A & $1.2 \times 10^{11}$ & $2.3 \times 10^{10}$ \\
\hline \hline
\end{tabular}




\section{CONCLUSION}

In conclusion, we have demonstrated an InAs/GaSb type-II superlattice n-B-p photodetector grown on GaAs substrate which operates in the MWIR range $\left(\lambda_{50 \%}\right.$ cutoff $\left.=6.4 \mu \mathrm{m}\right)$. Temperature dependent dark current analysis shows the dominance of diffusion at temperature higher than $130 \mathrm{~K}$. At 77 $\mathrm{K}$, a low dark current density of $2.9 \times 10^{-5} \mathrm{~A} / \mathrm{cm}^{2}$ under $-0.1 \mathrm{~V}$ is achieved, corresponding to a $R_{0} A$ value in excess of $8 \times 10^{3}$ $\Omega \cdot \mathrm{cm}^{2}$. QE between 10 to $15 \%$ is observed at near cutoff wavelength at $77 \mathrm{~K}$ under zero bias, and the peak specific detectivity is $7.6 \times 10^{11} \mathrm{~cm} \cdot \mathrm{Hz}^{1 / 2} / \mathrm{W}$. Future works should be focused on the enhancement of $\mathrm{QE}$ and reduction of dark current, such as the fine-tuning of absorber thickness and the dislocation buffer layer, in order to compete with the state-of-the-art devices while enjoying the cost benefit of non-native substrates at the same time.

\section{REFERENCES}

W. Chen, Z. Deng, D. Guo, Y. Chen, Y. I. Mazur, Y. Maidaniuk, et al., "Demonstration of InAs/InGaAs/GaAs Quantum Dots-in-a-Well Mid-Wave Infrared Photodetectors Grown on Silicon Substrate," Journal of Lightwave Technology, vol. 36, pp. 2572-2581, 2018

[2] J. Huang, D. Guo, W. Chen, Z. Deng, Y. Bai, T. Wu, et al., "Sub-monolayer quantum dot quantum cascade mid-infrared photodetector," Applied Physics Letters, vol. 111, p. 251104, 2017.

[3] A. K. Sood, J. W. Zeller, R. E. Welser, Y. R. Puri, N. K. Dhar, P. S. Wijewarnasuriya, et al., "Design and Development of Two-Dimensional Strained Layer Superlattice (SLS) Detector Arrays for IR Applications," in Two-dimensional Materials for Photodetector, P. K. Nayak, Ed., ed, 2018.

[4] A. Rogalski, "Recent progress in infrared detector technologies," Infrared Physics \& Technology, vol. 54, pp. 136-154, 2011/05/01/ 2011

[5] E. GOMÓŁKA, O. MARKOWSKA, M. KOPYTKO, A. KOWALEWSKI, P. MARTYNIUK, A. ROGALSKI, et al., "Mid-wave InAs/GaSb superlattice barrier infrared detectors with $\mathrm{nBnN}$ and $\mathrm{pBnN}$ design," BULLETIN OF THE POLISH ACADEMY OF SCIENCES TECHNICAL SCIENCES, vol. 66, 2018.

[6] J. B. Rodriguez, E. Plis, G. Bishop, Y. D. Sharma, H. Kim, L. R. Dawson, et al., "nBn structure based on InAs / GaSb type-II strained layer superlattices," Applied Physics Letters, vol. 91, p. 043514, 2007.

[7] S. A. Pour, E. K. Huang, G. Chen, A. Haddadi, B. M. Nguyen, and M. Razeghi, "High operating temperature midwave infrared photodiodes and focal plane arrays based on type-II InAs/GaSb superlattices," Applied Physics Letters, vol. 98, p. 143501, 2011/04/04 2011.

[8] A. D. Hood, A. J. Evans, A. Ikhlassi, D. L. Lee, and W. E. Tennant, "LWIR Strained-Layer Superlattice Materials and Devices at Teledyne Imaging Sensors," Journal of Electronic Materials, vol. 39, pp. 1001-1006, July 012010.

[9] N. Gautam, H. S. Kim, M. N. Kutty, E. Plis, L. R. Dawson, and S. Krishna, "Performance improvement of longwave infrared photodetector based on type-II InAs/GaSb superlattices using unipolar current blocking layers," Applied Physics Letters, vol. 96, p. 231107, 2010.

[10] E. Plis, J. B. Rodriguez, G. Balakrishnan, Y. D. Sharma, H. S. Kim, T. Rotter, et al., "Mid-infrared InAs/GaSb strained layer superlattice detectors with $\mathrm{nBn}$ design grown on a GaAs substrate," Semiconductor Science and Technology, vol. 25, p. 085010, 2010.

[11] Z. Deng, B. Chen, X. Chen, J. Shao, Q. Gong, H. Liu, et al., "Optical properties of beryllium-doped GaSb epilayers grown on GaAs substrate," Infrared Physics \& Technology, vol. 90, pp. 115-121, 5// 2018 .
[12] A. Jallipalli, G. Balakrishnan, S. Huang, T. Rotter, K. Nunna, B. Liang, et al., "Structural Analysis of Highly Relaxed GaSb Grown on GaAs Substrates with Periodic Interfacial Array of $90^{\circ}$ Misfit Dislocations," Nanoscale Research Letters, vol. 4, p. 1458, August 302009.

[13] C. G. Burguete, D. Guo, P. Jurczak, F. Cui, M. Tang, W. Chen, et al., "Direct growth of InAs/GaSb type II superlattice photodiodes on silicon substrates," IET Optoelectronics, vol. 12, pp. 2-4, 2018.

[14] S. H. Huang, G. Balakrishnan, A. Khoshakhlagh, A. Jallipalli, L. R. Dawson, and D. L. Huffaker, "Strain relief by periodic misfit arrays for low defect density GaSb on GaAs," Applied Physics Letters, vol. 88, p. 131911, 2006.

[15] M. Kopytko and A. Rogalski, "HgCdTe barrier infrared detectors," Progress in Quantum Electronics, vol. 47, pp. 1-18, 2016/05/01/ 2016.

[16] Z. Taghipour, S. Lee, S. A. Myers, E. H. Steenbergen, C. P. Morath, V. M. Cowan, et al., "Temperature-Dependent Minority-Carrier Mobility in p-Type InAs/GaSb Type-II-Superlattice Photodetectors," Physical Review Applied, vol. 11, p. 024047, $02 / 19 / 2019$

[17] D. Guo, Q. Jiang, M. Tang, S. Chen, Y. I. Mazur, Y. Maidaniuk, et al., "Two-colour In0.5Ga0.5As quantum dot infrared photodetectors on silicon," Semiconductor Science and Technology, vol. 33, p. 094009, 2018/08/21 2018.

[18] B. Chen, W. Jiang, J. Yuan, A. L. Holmes, and B. M. Onat, "SWIR/MWIR InP-based PIN photodiodes with InGaAs/GaAsSb type-II quantum wells," IEEE Journal of Quantum Electronics, vol. 47, pp. 1244-1250, 2011.

[19] B.-M. Nguyen, D. Hoffman, E. K.-w. Huang, S. Bogdanov, P.-Y. Delaunay, M. Razeghi, et al., "Demonstration of midinfrared type-II InAs/GaSb superlattice photodiodes grown on GaAs substrate," Applied Physics Letters, vol. 94, p. 223506, 2009.

[20] A. Kazemi, S. Myers, Z. Taghipour, S. Mathews, T. Schuler-Sandy, $\mathrm{S}$. Lee, et al., "Mid-wavelength infrared unipolar nBp superlattice photodetector," Infrared Physics \& Technology, vol. 88, pp. 114-118, 2018/01/01/2018.

[21] B. Chen and A. L. Holmes, "InP-based short-wave infrared and midwave infrared photodiodes using a novel type-II strain-compensated quantum well absorption region," Optics letters, vol. 38, pp. 2750-2753, 2013.

[22] B. Chen, W. Jiang, J. Yuan, A. L. Holmes, and B. M. Onat, "Demonstration of a Room-Temperature InP-Based Photodetector Operating Beyond 3 m," IEEE Photonics Technology Letters, vol. 23, pp. 218-220, 2011.

[23] K. Melih, A. Bulent, Y. E. Suyolcu, A. Bulent, and S. Uğur, "Performance evaluation of $\mathrm{InAs} / \mathrm{GaSb}$ superlattice photodetector grown on GaAs substrate using AlSb interfacial misfit array," Semiconductor Science and Technology, vol. 33, p. 035002, 2018.

Zhuo Deng received the B.Sc. and Ph.D. degrees from the University of Hong Kong in 2010 and 2015, respectively. In 2015, he was a Research Associate with the Department of Physics, University of Hong Kong, where he has been engaged in research of multi-junction photovoltaic based on III-V compound semiconductors. In 2016, he joined the School of Information Science and Technology, ShanghaiTech University in Shanghai, China as a Research Fellow. His current research interests include optical properties of III-V compound semiconductors and design, fabrication, and characterization of infrared photodetectors based on III-V compound semiconductor nanostructures.

Daqian Guo received the Bachelor's degree in materials science and engineering with Swansea University, U.K. Since 2016, he has been working toward the Ph.D. degree at the Molecular Beam Epitaxy Group, University College London, London, U.K. His research interests include mid-wavelength infrared photodetectors and molecular beam epitaxy growth of III-V semiconductors.

Jian Huang received the B.S. degree in materials science and engineering from Xidian University, Xi'an, China, in 2017. He is currently working toward the Master's degree at the School of Information Science and Technology, ShanghaiTech University, Shanghai. His research interest is midwave infrared photodetectors. 
Huiyun Liu received the degree from the Institute of Semiconductor, Chinese Academy of Sciences, Beijing, China, in August 2001. In 2007, he was awarded Royal Society University Research Fellow and started his academic career with the Department of Electronic and Electrical Engineering, University College London, London, U.K., where he is currently a Professor of semiconductor photonics. He has co-authored more than 300 papers in the area of semiconductor materials and devices. His general interest concentrates on the nanometre-scale engineering of low-dimensional semiconductor structures (such as quantum dots, quantum wires, and quantum wells) by using molecular beam epitaxy and the development of novel optoelectronic devices including lasers, detectors, and modulators by developing novel device process techniques.

Jiang $\mathbf{W u}$ received the B.S. degree from the University of Electronic Science and Technology of China (UESTC), Chengdu, China, and the M.Sc. and Ph.D. degrees in electrical engineering from the University of Arkansas, Fayetteville, AR, USA, in 2008 and 2011, respectively. After the Ph.D. degree, he joined the State Key Laboratory of Electronic Thin Films and Integrated Devices, UESTC. From 2012 to 2018, he worked at the Photonics Group in University College London, UK, focusing on Molecular Beam Epitaxy of III-V compound semiconductors and optoelectronic devices. He is currently a professor at UESTC.

Baile Chen received the Bachelor's degree in physics from the Department of Modern Physics, University of Science and Technology of China in Hefei,

China, in 2007. He received the Master's degree in physics and the Ph.D. degree in electrical engineering from the University of Virginia, Charlottesville, VA, USA, in 2009 and 2013, respectively. In February of 2013, he joined the Qorvo Inc., Hillsboro, OR, USA, as an RF product development engineer working on various RF power amplifiers and BAW filters for RF wireless communication systems. In January 2016, he joined the School of Information Science and Technology, Shanghai Tech University, Shanghai, China, as a Tenure Track Assistant Professor, PI. His research interests include III-V compound semiconductor materials and devices, silicon photonics. 